\title{
P01-85
}

\section{ASSOCIATION BETWEEN OREXIN PLASMA CONCENTRATION AND PSYCHOPATHOLOGY IN ALCOHOL DEPENDENT PATIENTS DURING ACUTE DETOXIFICATION}

\author{
C. von der Goltz ${ }^{1}$, M. Grosshans ${ }^{1}$, A. Koopmann ${ }^{1}$, T. Fink ${ }^{2}$, K. Wiedemann ${ }^{2}$, F. Kiefer ${ }^{1}$ \\ ${ }^{1}$ Central Institute of Mental Health, Mannheim, ${ }^{2}$ Department of Psychiatry, University Hospital of Hamburg, Hamburg, Germany
}

\begin{abstract}
Aims: The orexins (hypocretins) are neuropeptides recently identified as neurotransmitters in lateral hypothalamus neurons. Although the majority of studies associated the function of orexin neurons with arousal and sleep, these neurons also project to reward-associated brain regions, including the nucleus accumbens and ventral tegmental area. This indicates a possible role for orexins in reward function and motivation and thus in addictive diseases. Additionally, there is growing evidence from preclinical studies for an involvement of orexins in the regulation of stress, affectivity and drug seeking behavior.

Method: We investigated orexin plasma concentrations and psychological symptoms in a sample of 34 alcohol dependent subjects on day 1 and day 14 of detoxification. For this purpose we used the Brief Symptom Inventory (BSI) as well as the ObsessiveCompulsive Drinking Scale to identify self-reported clinically relevant psychological symptoms including alcohol craving.
\end{abstract}

Results: As a main result a significant positive correlation between orexin plasma concentration and depression as well as global distress indicies of the BSI was detected during early withdrawal (day 1), which is not shown after detoxification on day 14. No association with subjective craving for alcohol was found.

Conclusion: Our data indicate that orexins may be directly involved in affective dysregulation in alcohol dependent patients; moreover the effects of orexins on reinstatement of drug seeking behaviors might be mediated by impaired brain stress systems. 